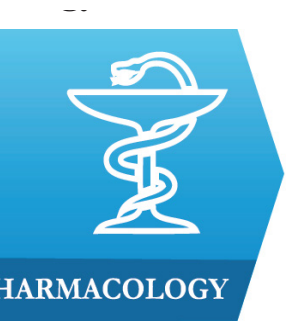

1) Faculty of Pharmacy, Iuliu Hațieganu University of Medicine and Pharmacy, Cluj-Napoca, Romania

2) Centro de Investigação em Saúde e Ambiente, Escola Superior de Saúde, Instituto Politécnico do Porto, Porto, Portugal

\section{Safety and efficacy of iota-carrageenan nasal spray in treatment and prevention of the common cold}

\author{
Diana Bichiri ${ }^{1}$, Ana Rita Rente ${ }^{2}$, Ângelo Jesus ${ }^{2}$
}

DOI: $10.15386 / \mathrm{mpr}-1817$

Manuscript received: 18.07.2020

Received in revised form: 16.10.2020

Accepted: 02.11.2020

Address for correspondence:

dianabichiri@gmail.com

This work is licensed under a Creative Commons Attribution-NonCommercialNoDerivatives 4.0 International License

\begin{abstract}
The common cold is one of the most frequent viral infections in humans. Although benign, its symptoms result in economic burden and can lead to severe or even fatal complications in children, elderly and groups with comorbidities. The main purpose of the treatment is the relief of symptoms; however, the medication is often associated with adverse effects. Iota-carrageenan is a polysaccharide that reveals antiviral activity by binding to viruses, inhibiting its replications and, consequently, its viral propagation. This systematic review of the literature aims to compare the effectiveness of an iota-carrageenan nasal spray to placebo. This systematic review was conducted through research in Cochrane Database, PubMed, Science Direct, SpringerLink, Oxford Journals, Elsevier, ClinicalKey, Wiley Online Library, Embase databases, in order to collect randomized and controlled clinical trials. In total, the research provided four articles regarding clinical trials for comparing iota-carrageenan nasal spray with placebo. The results show it has potent antiviral activity compared to placebo and a favorable safety profile. Although further research is needed, the concept of a physical barrier capable of reducing viral penetration of epithelial cells in the nasal mucosa is appealing, and could lead to alternative approaches, with positive impact on global health.
\end{abstract}

Keywords: carrageenan, iota-carrageenan, common cold, safe treatment, antiviral

\section{Introduction}

The common cold is defined as a spontaneously remitting acute viral infection of the upper respiratory tract involving the nose, sinuses, pharynx and larynx $[1,2]$. It is characterized by symptoms that are indistinguishable among different viral pathogens like nasal stuffiness and discharge, sore throat, cough, malaise and sometimes fever (usually below $37.8^{\circ}$ C) $[3,4]$. The common cold is one of the most frequent, usually mild, human disease that can significantly affect the quality of life and public health, having a negative economic impact due to frequent health care services needed and absences from school or work $[5,6]$.

The etiology of common cold is linked to the infection with human rhinoviruses (HRVs), respiratory syncytial virus (RSV), influenza and parainfluenza viruses, coronaviruses and adenoviruses. The particular virus which is responsible for $50-80 \%$ of common colds and $30-50 \%$ of all respiratory illnesses is HRV, which often causes loss of productivity, misuse of antibiotics and increased morbidity. Some viruses have wide variations of infections during a year: coronaviruses infections usually happen in winter or early spring, influenza virus between November and March, parainfluenza and HRV during autumn $[5,6]$. One of the most difficult parts in the development of an antiviral treatment for the common cold is the wide range of pathogens. Focusing only on a specific virus family would be effective in treating only a certain amount of all colds, being an unsuitable approach for a general therapy. This particular limitation is one of 
the disavantages of antiviral medicines that block the viral receptor or host cell receptor [7].

The pathogenesis of the common cold consists of a complex set of actions, including the replication of the virus in the host cells and the inflammatory response caused by infection. Although it is commonly known that viruses infect the upper respiratory tract, some viruses have very particular anatomical sites where they replicate: influenza viruses in the tracheobronchial epithelium and rhinoviruses usually in the nasopharynx. The first contact of rhinovirus in humans is on the anterior nasal mucosa or the eye, from where they are transported to the posterior nasopharynx by mucociliary motion. After that, viruses bind with specific serotype-dependent receptors: about $90 \%$ use the intracellular adhesion molecule-1 (ICAM-1) receptor for HRV major serotypes, while some use the low-density lipoprotein (LDL) receptor for HRV minor serotypes. After the penetration of epithelial cell, the virus starts to multiply very quickly and triggers inflammatory responses: vasodilatation which produces nasal obstruction and increased vascular permeability responsible for rhinorrhoea. The cholinergic receptors are stimulated and cause sneezing and increased mucous gland secretions $[5,6]$.

The clinical diagnosis is usually simple and the adult patients often self-diagnose reliably, but there are some limitations on children based on their incapacity of expressing their symptoms. In order to identify the exact type of virus that caused the problem, the most accurate methods of diagnosis include viral culture, antigen detection and polymerase chain reaction (PCR). The main advantages of PCR include the sensitivity, specificity, high detection rate, cost-effectiveness, subtyping capability to new viruses, making it the method of choice among other virus detection techniques in upper respiratory tract infections. Nasopharyngeal aspirates and nasal wash are the optimal collection techniques, but nasal and throat swabs are also used because of their great feasibility $[5,6]$.

As stated before, the common cold presents an extraordinary challenge for the development of antiviral treatments because of the diversity of viruses that can be implicated. However, there is one factor shared by all the common cold viruses: they must somehow reach their specific host cell receptor. Providing some sort of mechanical barrier could be an effective strategy [7].

A new approach with high antiviral potential could be the iota-carrageenan intra-nasal spray for treating the common cold. Carrageenan is a generic name for a family of polysaccharides, obtained from certain species of red seaweeds of the class Rhodophyceae. Carrageenans are sulfated polygalactans with a molecular weight higher than $100 \mathrm{kDa}$. Carrageenan is classified into various subtypes; the three main copolymers are iota-, kappa- and lambdacarrageenan [8]. Carrageenan copolymers are widely used in cosmetic products and in the food industry. They are also increasingly used in the pharmaceutical industry e.g. as emulsifying and viscosifying agents in various topical products [9].

It has been demonstrated that iota-carrageenan exerts antiviral activity against HRVs and influenza viruses [10], herpes simplex virus (HSV), cytomegalovirus (CMV), dengue virus, human papilloma virus (HPV), and human immunodeficiency virus (HIV) [11-14]. The previous literature suggests that the antiviral effects of iotacarrageenan are not mediated by interaction with the host but by building a physical barrier preventing binding and / or entry of the virus into the cells [7].

In this paper we aim to analyze the current literature regarding the application of iota-carrageenan nasal spray for the common cold, by reviewing existing scientific literature about the efficacy and safety compared to placebo.

\section{Methodology}

As a systematic review, this text is structured as a synthesis of all the results provided by the eligible clinical trials [15]. Our question was if the administration of a nasal spray containing iota-carrageenan may be a safe and efficient alternative for treating the common cold. We applied the Population, Intervention, Comparison and Outcome (PICO) for the research strategy, as follows:

- Population: individuals (children and adults) with mild symptoms at the early stages of the common cold

- Intervention: administrations of Iota-Carrageenan (IC) $0.12 \%$ nasal spray (Marinomed Biotechnologie)

- Comparison: placebo

- Outcome: variations of mean total symptom score (TSS) after the intervention, quantitative determination of viral load from nasal wash samples by reverse transcription polymerase chain reaction (RT-PCR) method, safety parameters during trial (side effects).

The keywords used were ,iota-carrageenan”, „,carrageenan”, „, nasal spray”, „,common cold”, „placebo” used in various combinations. Also, when applicable, the filter "clinical trial" was applied.

In order to explore the existing clinical studies, we used the following recommended online databases: Cochrane Database, PubMed, Science Direct, SpringerLink, Oxford Journals, Elsevier, ClinicalKey, Wiley Online Library, Embase. The documenting process was accomplished in May 2020 and the language used for searching documents was English.

In order to assess the efficacy of IC nasal spray regarding the symptoms of common cold, the primary variable we investigated was the difference of Total Symptoms Score (TSS) compared to placebo. TSS is a scale used by clinical researchers to appreciate the patient's perceptions of symptom intensity in the common cold. The scale is meant to evaluate eight clinical features including systemic symptoms (SSS) (headache, chilliness, muscle 
ache) and local symptoms (LSS) (sore throat, runny or blocked nose, cough, sneezing). The patient must score each symptom from 0 (absent) to 1 (mild), 2 (moderate) or 3 (severe) [16].

The second variable we analyzed was the quantitative determination of viral load from nasal mucosa, collected before and during the treatment with IC nasal spray, compared to placebo.

The intensity and severity of the side effects that occurred were two important factors in determining the safety profile.

\section{Results}

The result of the documenting process is available in figure 1. We used the PRISMA flow diagram in order to summarize our findings. During this process, only one article was excluded because it was based on the clinical trials we already included.

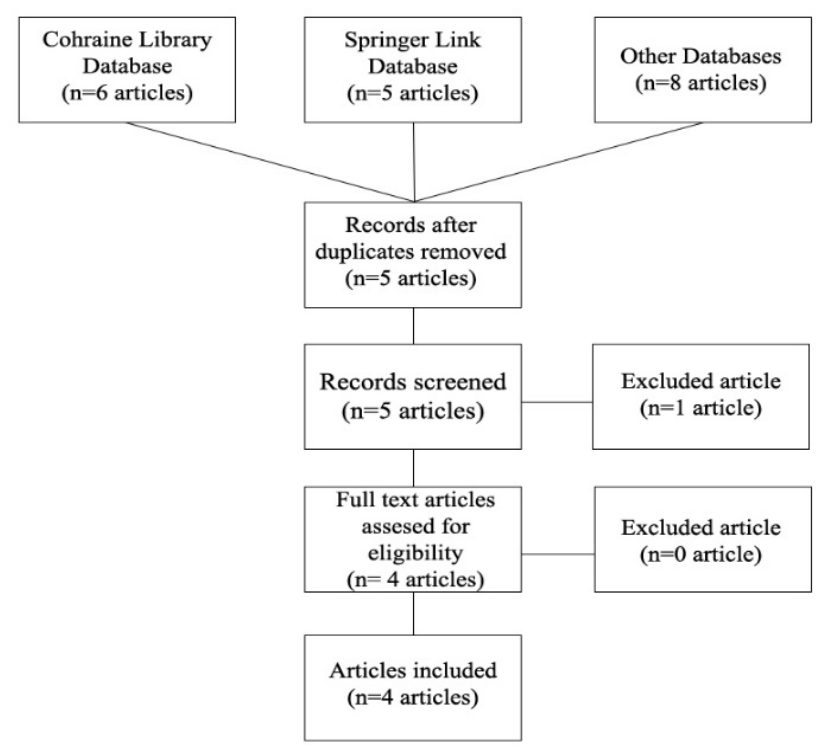

Figure 1. PRISMA diagram flow results.

Table I. Jadad scale applied on clinical trials that passed PRISMA selection.

\begin{tabular}{l|c|c|c|c|} 
Question & Eccles et al. & Fazekas et al. & Ludwig et al. 2013 & Eccles et al. 2015 \\
[19] & $\mathbf{2 0 1 0}[\mathbf{1 8}]$ & $\mathbf{2 0 1 2}[\mathbf{1 6}]$ & $+1 \mathrm{p}$ & $+1 \mathrm{p}$ \\
\hline Was the study described as randomized? & $+1 \mathrm{p}$ & $+1 \mathrm{p}$ & $+1 \mathrm{p}$ & 0 \\
Was the method of randomization appropriate? & $+1 \mathrm{p}$ & $+1 \mathrm{p}$ & $+1 \mathrm{p}$ & $+1 \mathrm{p}$ \\
Was the study described as blinding? & $+1 \mathrm{p}$ & $+1 \mathrm{p}$ & $+1 \mathrm{p}$ & $+\mathrm{p}$ \\
Was the method of blinding appropriate? & $+1 \mathrm{p}$ & $+1 \mathrm{p}$ & $+1 \mathrm{p}$ & $\mathbf{5 p}$
\end{tabular}

Table II. The results and characteristics of the included clinical trials.

\begin{tabular}{|c|c|c|c|c|}
\hline Clinical trial & Eccles et al. 2010 [18] & Fazekas et al. 2012 [16] & Ludwig et al. 2013 [19] & Eccles et al. 2015 [20] \\
\hline Patients & 35 adults, mean age 19.6 years & 153 children, mean age 5 years & 200 , mean age 20 years & 211 , mean age 33 years \\
\hline $\begin{array}{l}\text { TSS } \\
\text { differences }\end{array}$ & $\begin{array}{l}\text { - Lower TSS on days } 2-4 \\
(p=0.046) \\
\text { - Lower LSS on days } 2-4 \\
(p=0.064) \\
\text { - Lower mean score for } \\
\text { blocked nose on day } 7(0.42 \\
\text { vs } 0.78)\end{array}$ & $\begin{array}{l}\text { Non-significant reduction on } \\
\text { days } 2-7 \text { ( } 4.0 \text { vs } 3.8)\end{array}$ & $\begin{array}{l}\text { - Non-significant differences } \\
\text { during early phase of disease } \\
\text { - Significant reduction in the } \\
\text { later days of the disease }(0.56 \text {, } \\
0.55 \text { vs } 0.24,0.23 ; \mathrm{p} 1=0.029 \text {, } \\
\text { p2 }=0.048)\end{array}$ & $\begin{array}{l}\text { Significant reduction } \\
\text { between days } 2-4(5.67 \text { vs } \\
6.39 ; p=0.0364)\end{array}$ \\
\hline Viral load & Decreased by $92 \%(\mathrm{p}<0.009)$ & $\begin{array}{l}\text { Decreased significantly } \\
\text { between days } 3-5(p=0.026) \\
\text { - lower incidence of secondary } \\
\text { infections }(13 \% \text { vs } 27 \% \text {, } \\
p=0.046) \\
\text { - higher rates of clearing the } \\
\text { viruses }(52.2 \% \text { vs } 32.4 \% \text {, } \\
p=0.030)\end{array}$ & $\begin{array}{l}\text { Decreased significantly on } \\
\text { days } 3-4\left(\log _{10} \text { viral titer } 2.15 \text {, }\right. \\
2.19 \text { vs } 1.38,1.37 ; \mathrm{p} 1=0.024, \\
\text { p2=0.018) }\end{array}$ & $\begin{array}{l}\text { Decreased on days } 3-4 \\
(90.2 \% \text { vs } 72 \% \\
p=0.0958)\end{array}$ \\
\hline Side effects & $\begin{array}{l}\text { Puffy eyes, dry mouth. } \\
\text { Good safety profile }\end{array}$ & Good safety profile & Good safety profile & $\begin{array}{l}\text { Migraine, malaise, } \\
\text { toothache. } \\
\text { Good safety profile }\end{array}$ \\
\hline Observations & $\begin{array}{l}\text { Reduced pro-inflammatory } \\
\text { cytokines }\end{array}$ & $\begin{array}{l}\text { - Reduction of symptoms } 1.8 \\
\text { days faster ( } 7.6 \text { vs } 9.4 \text { days, } \\
p=0.038)\end{array}$ & $\begin{array}{l}\text { - Reduction of symptoms } 2.1 \\
\text { days faster }(\mathrm{p}=0.037) \\
\text { - Earlier breakpoint }(3.4 \text { vs } 3.9 \\
\text { days, } \mathrm{p} 1=0.025, \mathrm{p} 2=0.064)\end{array}$ & $\begin{array}{l}\text { Non-significant reduction } \\
\text { of symptoms duration }(7.5 \\
\text { vs } 7.4 \text { days) }\end{array}$ \\
\hline
\end{tabular}

Legend: TSS: total score symptom; LSS: local score symptom; vs: versus; First number: iota-carrageenan group versus Second number: placebo group. 
In order to review the quality of the selected clinical trials, we used the Jadad scale to appreciate the randomization, blinding and the presence of final records for each individual. Each question might have a "yes" or "no" answer, which is pointed +1 for yes, 0 for the lack of information and -1 for "no". The Jadad scale is based on 5 questions, therefore the range of points might vary from -5 to +5 , considering that a score of lower than 3 means low quality of the trial [17]. The results of our selected trials are summarized in table I.

\section{Study characteristics}

Table II summarizes the characteristics of all 4 studies included in this review. A total of 599 patients were involved.

\section{Clinical efficacy regarding TSS variations}

The study conducted by Eccles and his team in 2010 was designed as an exploratory trial, the size of the study was relatively small (34 participants) but reached statistical significance for the predefined primary endpoint (TSS mean of sum on days 2-4). However, the efficacy of the Iota-Carrageenan nasal spray treatment appeared to be mainly dependent on the LSS: sore throat, blocked nose, runny nose, cough, and sneezing. It is important to mention that the medication was applied only for the first 4 days. This could be a reason why a complete relief of symptoms did not occur. Another important finding in this study is that the pro-inflammatory mediators FGF-2, Fractalkine, GRO, G-CSF, IL-8, IL-1a, IP-10, IL-10, and IFN-a2 were lower in the IC group. This result might be correlated with the decreased viral replication and therefore, with the lower TSS. The reduction of the blocked nose score symptoms in the IC group corresponds to a $50 \%$ decrease compared to the first days of study [18].

In a study from 2012 of 153 pediatric patients with ages between 1-18 years, the researchers analyzed and compared the mean TSS values of days 2-7 from both groups. No significant differences were observed on days 2-7 of the study between placebo and IC nasal spray. The authors point out that these results might have been influenced by the difficulty in assessing the symptom scores of young children. Nevertheless, the researchers showed that the mean time to persistent symptoms was significantly shorter in the group treated with IC nasal spray. Therefore, a reduction of symptoms earlier than placebo was demonstrated during this trial in pediatric patients [16].

The results of a clinical trial conducted in 2013, including 211 participants, are comparable with the previous studies discussed. The authors did not find any significant difference comparing the mean of TSS scores from the IC and the placebo groups in the first phases of the disease. Although the first 7 days of comparison showed no major differences, the results over this period of time showed that the IC groups presented faster reduction of symptom intensity compared to placebo. The breakpoint was defined as the point where the disease changes its course and it occurred earlier in both IC groups. This study demonstrated that the patients who used IC nasal spray presented an alleviation of symptoms earlier compared to placebo [19].

Another clinical trial from 2015 included approximately 200 volunteer patients and split them into two groups: treated with IC and placebo. Patients in both groups had similar baseline of TSS. However, further exploratory analyses after unblinding (excluding a patient with aberrant high symptom scores of TSS) demonstrated treatment differences in favor of IC on days 2-4 of treatment. The mean time of disease symptom presence was almost the same in both groups, meaning no statistical difference [20].

\section{Viral load efficacy}

Eccles et al. observed in 2010 that patients treated with topical IC nasal spray presented dramatically lower viral load, being decreased almost totally. The placebo group presented with a major increase in the nasal viral load by almost 6 times, representing 579\% [18].

The clinical trial conducted by Fazekas et al. from 2012 showed that topical applications of IC nasal spray had beneficial effects regarding the viral load. The reduction of viral load was significant compared to placebo after 3-5 days. Also, around half of patients from the IC group cleared their viruses by the second visit (three to five days into treatment). The results from the second visit showed that patients from the IC group had a lower virus number from $\log _{10}$ viral load 1.3 to 0 , compared to the placebo group which did not present any significant difference. Another important finding is that the incidence of new infections was significantly lower in the IC group compared to placebo [16].

Ludwig et al. presented in the study from 2013 that patients from two IC groups had lower viral loads compared to two placebo groups. The median decrease of $\log _{10}$ viral titer was higher for both IC groups [19].

In the next study from 2015, Eccles et al. analyzed the viral load from nasal mucosa only from the virus-positive participants. Although only $54.9 \%$ of the population tested virus-positive, the viral load results suggest a trend towards greater reduction in IC group [20].

\section{Safety and tolerability}

In the first clinical trial from 2010, Ron Eccles et al. observed only two adverse reactions that were correlated with the administration of IC nasal spray. One patient reported dry mouth and another one puffy eyelids, both from the IC group. Considering the fact that these few side effects were resolved without any special actions, the researchers concluded that the IC nasal spray has a good safety profile [18].

In the 2012 study, Fazekas et al. reported that the presence of drug-related adverse reactions was lower in the IC group compared to placebo. The side effects consisted in upper respiratory tract symptoms for the most parts. This clinical trial concluded that both nasal sprays, IC and placebo, were well-tolerated [16]. 
Ludwig et al. observed in 2013 only one side effect related to treatment: a patient from the placebo group reported a burning feeling in the nose. Although there were a number of 43 side effects, only the one from the above was relevant, while the rest of them were not drug-related, having the same frequency in both groups. Therefore, the IC nasal spray was considered well tolerated [19].

The second clinical trial from 2015 conducted by Eccles et al. reported an incidence of 3\% adverse reactions of severe intensity in the IC group: one patient with headache and migraine, another patient with toothache and one patient with malaise. Despite the presence of these side effects, only 1 patient assessed the tolerability of treatment as poor, while $88.8 \%$ scored it as excellent, very good or good. Therefore, the researchers concluded that the IC nasal spray is safe and well tolerated [20].

\section{Discussion}

Common cold is a disease that often affects the general population of any age, having an important economic impact. Although it is a self-limited disease, it can be deadly on certain subgroups of the population, including the elderly and people with comorbidities. Due to its high variability of pathogens, the currently available treatments are only symptomatic with only few specific alternatives. Iota-carrageenan nasal spray could be a good non-specific alternative of treatment due to its capacity to block viral replication via a mechanical mechanism. Therefore, Iota-carrageenan might be a new approach because of its antiviral activity $[2,5]$.

The clinical trials tried to determine the efficacy and safety of IC nasal spray regarding common cold symptoms and duration of illness, using the IC nasal spray from 3 to 4 times per day. The main efficacy outcomes of the included studies were significant variations of TSS, duration of disease and viral load differences. The safety characteristics were assessed by the presence or absence of side effects related to the medicine. When a certain side effect occurred, the researchers analyzed the importance and the severity of it.

Although all the reviewed clinical researches had their limitations, only two of them (Eccles et al. in 2010 and 2015) succeeded to ascertain the efficacy of iota-carrageenan nasal spray in common cold symptoms regarding significant TSS variations in the first phase of illness.

Eccles et al. demonstrated in 2010 in a small (34 participants), double-blind, placebo-controlled clinical trial, statistical and significant improvements regarding common cold symptoms in the first days of disease after the administration of the IC nasal spray. The primary endpoint for efficacy was the differences of TSS from days $2-4$, having the IC group better results than placebo. The improvements were mainly observable on the LSS (more evident on the blocked nose) and little difference for SSS.
The specific efficacy on the blocked nose might be one of the reasons the IC nasal spray had higher acceptability in patients. Although no patient reported complete relief of symptoms, the authors reported that it may be because the IC nasal spray was administered for only 4 days. A longer period of treatment and observation might highlight better the efficacy of IC nasal spray. Compared to other antiviral specific treatments, the early intervention is correlated with high levels of efficacy, explaining why the participants of the clinical trial were included after 1 day or less from the start of the symptoms.

This is the only clinical trial that analyzed the quantitative determination of pro-inflammatory cytokines. There is clear evidence that the concentration is lower in the IC group, suggesting that the inflammation caused by the infection is milder, probably because of a lower rate of viral load.

At the beginning of the trial, some patients from the IC group reported higher scores on blocked nose, cough and sore throat. Interestingly, these symptoms worsened in the placebo group but not in the IC group, suggesting that administration of IC nasal spray could inhibit the development of common cold symptoms shortly after starting the treatment. This study did not analyze the effect of IC nasal spray on the duration of disease, compared to the other 3 studies we included. The results of this study should be viewed with caution due to the small number of patients [18].

The trial conducted by Eccles et al. in 2015 had, at first, two of three efficacy outcomes not statistically relevant: variations of TSS score from days 2-4 and duration of disease were similar in both studied groups. One of the most significant issues that affected this study was the inclusion of a patient who reported very high TSS scores and very different from others, possibly because of misinterpretation of the methodology. The authors reevaluated the results after excluding this patient and the TSS variations of days 2-4 became statistically significant between IC and the placebo group, similar to the clinical trial conducted in 2010. Another interesting aspect was that the authors presumed that the IC nasal spray might have had a very early effect on common cold symptoms. Instead of days 2-4, the authors analyzed the TSS scores from day 1-4 and the differences were statistically significant comparing both groups. In spite of the other 2 clinical that observed variations of TSS in the second phase of the disease trials (Fazekas et al. 2012, Ludwig et al. 2013), this study did not record any significant improvement in TSS after day 4-5 and therefore, no faster alleviation of symptoms [20].

The clinical study that included pediatric patients conducted by Fazekas et al. in 2012 observed a faster alleviation of symptoms by 1.8 days, comparable with the results obtained by Ludwig et al. in 2013. The use of IC nasal spray in children might be a beneficial alternative 
due to the fact that decongestionnant nasal sprays are not recommended in patients below 12 years. The main outcome of this study was the variation of the TSS mean from days 2-7 but there were not statistically significant differences, similar with the results obtained by Ludwig et al. in 2013. Another interesting finding was that a larger number of patients from the IC group tested negative for viruses in the second visit, being the only study that analyzed this particular aspect. This might mean that administration of IC nasal spray may help prevent recurrent upper respiratory system infections. Another different approach of this study is the analysis of the presence or absence of newly acquired viruses, suggesting that IC nasal spray could prevent co-infections. Some particular limitation might have been the difficulty of interpreting symptoms in very young patients like headache, muscle ache or chilliness. The use of co-medication (antipyretic and antitussive medication) might have been affecting the TSS results as well [16].

Although Ludwig et al. 2013 did not observe any statistical TSS differences between both groups in the early stages of disease, they mentioned some significant variations that occurred in the second phase of the illness resulting a faster alleviation of symptoms by 2.1 days. Patients treated with the IC nasal spray presented faster improvement of common cold symptoms because of the local antiviral activity of IC, but the duration of the disease was similar in both groups. In this study, the primary endpoint was the duration of disease, which was not statistically relevant when comparing the IC group to placebo. A significant limitation of this study is that only $58 \%$ of the included population tested virus-positive [19].

It is well known that iota-carrageenan nasal spray provides antiviral protection by a mechanical mechanism in vivo and in vitro. The clinical trials used the PCR method for determining the quantitative viral load from nasal lavages. As the previous in vivo studies suggested, the antiviral activity of the nasal spray is demonstrated by the results of 3 from 4 clinical trials reviews. The results also showed that administration of iota-carrageenan nasal spray can reduce the viral load of the nasal mucosa by $92 \%$, while in the placebo group it increased by around 5 times [10,16,18-21].

The significant antiviral activity is correlated with the mechanical antiviral properties of IC. This polysaccharide is a highly negative molecule that can interfere with the electrostatic approach of the positive charged virus to the host cell. At the beginning of infection, adjacent epithelial cells are being infected by newly produced viruses, causing higher viral loads and a deeper infection. If the IC is present during this phase, it acts similar to airway mucus and may trap viruses. The presence of both IC and airway mucus results in a strongly negative surface that can retain the newly produced positive charged viruses, preventing the expansion of the infection. The mucocilliary clearance will carry the mix of mucus, IC and trapped viruses into the nasopharynx, where it will be swallowed. The viruses end up being destroyed by the acid environment from the stomach [7].

Regarding the safety profile, all clinical trials have concluded that iota-carrageenan is a safe alternative. Although several side effects were reported, including few severe ones, there was no correlation with the medicine administered. The side effects occurred in the IC group and placebo group as well. There were only 2 adverse reactions that were concluded as drug-related: puffy eyes and dry mouth. During all 4 clinical trials, only 2 patients reported severe side effects from the IC group and 2 from the placebo group (from the same study), while the other 3 studies had no severe side effects at all. Therefore, all 4 clinical trials demonstrated that iota-carrageenan nasal spray is a safe alternative for treating the common cold $[16,18-20]$.

IC is made of a long polysaccharide chain that makes it unable to be absorbed from the nasal mucosa. The mechanical mechanism and the absence of pharmacological activity is correlated with the lack of known side effects and the lack of interactions with any drugs. The four placebo-controlled clinical trials confirm that there are no significant adverse effects related to the administration of IC nasal spray. Although there are no long-term studies regarding the safety, IC nasal spray has been authorized for several years without any safety concerns [7]. Since carrageenan has been used as a food additive for decades, there is an extended data set of safety and toxicity upon oral exposure [22]. Safety and toxicity of native iota-carrageenan after intranasal administration, has also been determined in non-clinical studies [8].

After analyzing all the results from the selected 4 studies, we believe that iota-carrageenan nasal-spray is a potent antiviral local treatment that can be useful treating the common cold, based on the favorable results of viral load analysis from 4 out of 4 studies. Although its clinical benefits regarding the symptoms or the duration of disease are not fully acknowledged, we believe that iota-carrageenan nasal spray is a promising alternative to treat common cold that requires more investigations on this aspect. Because of the favorable safety profile and mechanical mechanism of action, iota-carrageenan nasal spray might be a very useful alternative for particular physiological states of health (for example in pregnancy, older patients, young children, etc.).

A notable limitation of all studies might be the use of saline solution. Although it is a generally accepted option that leads to satisfactory results, there is limited evidence that the administration of saline solution intranasally might have benefits on upper respiratory tract infections [18]. Therefore, the saline IC solution $(0.5 \% \mathrm{NaCl}, 0.12 \%$ IC) and $0.9 \%$ saline solution that were used in all four clinical trials might limit the presented results. 


\section{Conclusions}

This systematic review regarding the safety and the efficacy of iota-carrageenan nasal spray for treating the common cold suggests that there is a need for further investigation to clearly demonstrate the efficacy in treating symptoms. While the results regarding the duration of symptoms are not definitive, some promising studies are suggesting that the IC nasal spray could reduce them by 1.8-2.1 days. The safety profile of iota-carrageenan makes it a safe alternative with very few side effects and high tolerability. Extensive clinical trials are still needed to have a solid scientific background for the efficacy and safety of iota-carrageenan nasal spray treating the common cold, however, the concept of a physical barrier capable of reducing viral penetration of epithelial cells in the nasal mucosa is appealing and could lead to alternative approaches, with a positive impact on global health.

\section{References}

1. Allan MG, Arroll B. Prevention and treatment of the common cold: making sense of the evidence. CMAJ. 2014;186:190199.

2. Simancas-Racines D, Franco JV, Guerra CV, Felix ML, Hidalgo R, Martinez-Zapata MJ. Vaccines for the common cold. Cochrane Database Syst Rev. 2017;5: CD002190.

3. Eccles R. Understanding the symptoms of the common cold and influenza. Lancet Infect Dis. 2005;5:718-725.

4. Wat D. The common cold: a review of the literature. Eur J Intern Med. 2004;15:79-88.

5. Heikkinen T, Järvinen A. The common cold. Lancet. 2003;361:51-59.

6. Passioti M, Maggina P, Megremis S, Papadopoulos NG. The common cold: potential for future prevention or cure. Curr Allergy Asthma Rep. 2014;14:413.

7. Eccles R. Iota-Carrageenan as an Antiviral Treatment for the Common Cold. Open Virol J. 2020;14:9-15.

8. Hebar A, Koller C, Seifert JM, Chabicovsky M, Bodenteich A, Bernkop-Schnürch A, et al. Non-clinical safety evaluation of intranasal iota-carrageenan. PLoS One. 2015;10:e122911.

9. Li L, Ni R, Shao Y, Mao S. Carrageenan and its applications in drug delivery. Carbohydr Polym. 2014;103:1-11.

10. Grassauer A, Weinmuellner R, Meier C, Pretsch A, PrieschlGrassauer E, Unger H. Iota-Carrageenan is a potent inhibitor of rhinovirus infection. Virol J. 2008;5:107.
11. González ME, Alarcón B, Carrasco L. Polysaccharides as antiviral agents: antiviral activity of carrageenan. Antimicrob Agents Chemother. 1987;31:1388-1393.

12. Baba M, Snoeck R, Pauwels R, de Clercq E. Sulfated polysaccharides are potent and selective inhibitors of various enveloped viruses, including herpes simplex virus, cytomegalovirus, vesicular stomatitis virus, and human immunodeficiency virus. Antimicrob Agents Chemother. 1988;32:1742-1745.

13. Talarico LB, Pujol CA, Zibetti RG, Faría PC, Noseda MD, Duarte ME, et al. The antiviral activity of sulfated polysaccharides against dengue virus is dependent on virus serotype and host cell. Antiviral Res. 2005;66:103-110.

14. Buck CB, Thompson CD, Roberts JN, Müller M, Lowy DR, Schiller JT. Carrageenan is a potent inhibitor of papillomavirus infection. PLoS Pathog. 2006;2:e69.

15. Higgins JP, Green S. Cochrane Handbook for Systematic Reviews of Interventions. The Cochrane Collaboration John Wiley \& Sons Ltd. 2008.

16. Fazekas T, Eickhoff P, Pruckner N, Vollnhofer G, Fischmeister G, Diakos C, et al. Lessons learned from a double-blind randomised placebo-controlled study with a iota-carrageenan nasal spray as medical device in children with acute symptoms of common cold. BMC Complement Altern Med. 2012;12:147.

17. Cioffi I, Farella M. Quality of randomised controlled trials in dentistry. Int Dent J. 2011;61:37-42.

18. Eccles R, Meier C, Jawad M, Weinmüllner R, Grassauer A, Prieschl-Grassauer E. Efficacy and safety of an antiviral Iota-Carrageenan nasal spray: a randomized, double-blind, placebo-controlled exploratory study in volunteers with early symptoms of the common cold. Respir Res. 2010;11:108.

19. Ludwig M, Enzenhofer E, Schneider S, Rauch M, Bodenteich A, Neumann K, et al. Efficacy of a carrageenan nasal spray in patients with common cold: a randomized controlled trial. Respir Res. 2013;14:124.

20. Eccles R, Winther B, Johnston SL, Robinson P, Trampisch M, Koelsch S. Efficacy and safety of iota-carrageenan nasal spray versus placebo in early treatment of the common cold in adults: the ICICC trial. Respir Res. 2015;16:121.

21. Leibbrandt A, Meier C, König-Schuster M, Weinmüllner $\mathrm{R}$, Kalthoff D, Pflugfelder B, et al. Iota carrageenan is a potent inhibitor of influenza A virus infection. PLoS One. 2010;5:e14320.

22. McKim JM. Food additive carrageenan: Part I: A critical review of carrageenan in vitro studies, potential pitfalls, and implications for human health and safety. Crit Rev Toxicol. 2014;44:211-243. 